\title{
On Capacity of Cognitive Radio Networks with Average Interference Power Constraints
}

\author{
Cheng-Xiang Wang, Senior Member, IEEE, Xuemin Hong, Member, IEEE, \\ Hsiao-Hwa Chen, Senior Member, IEEE, and John Thompson, Member, IEEE
}

\begin{abstract}
Cognitive radio (CR) has been considered as a promising technology to improve the spectrum utilization. In this paper we analyze the capacity of a CR network with average received interference power constraints. Under the assumptions of uniform node placements and a simple power control scheme, the maximum transmit power of a target CR transmitter is characterized by its cumulative distribution function (CDF). We study two CR scenarios for future applications. The first scenario is called the CR based central access network, which aims at providing broadband access to $C R$ devices. In the second scenario, the so-called $\mathrm{CR}$ assisted virtual multiple-input multiple-output (MIMO) network, CR is used to improve the access capability of a cellular system. The uplink ergodic channel capacities of both scenarios are derived and analyzed with an emphasis on understanding the impact of numbers of primary users and CR users on the capacity. Numerical and simulation results suggest that the CR based central access network is more suitable for less-populated rural areas where a relatively low density of primary receivers is expected; while the CR assisted virtual MIMO network performs better in urban environments with a dense population of mobile $C R$ users.
\end{abstract}

Index Terms-Cognitive radio network, network capacity, interference power constraint, virtual MIMO.

\section{INTRODUCTION}

$\mathbf{R}$ ADIO frequency spectrum is a precious resource which has become increasingly scarce due to wide deployment of wireless services. However, current license-based spectrum management policy has resulted in a low spectrum utilization [1]. The imbalance between spectrum scarcity and low spectrum utilization motivates the development of innovative spectrum sharing technologies to improve the spectrum utilization. As a promising spectrum sharing technology, cognitive radio (CR) enables a secondary network to dynamically

Manuscript received September 28, 2007; revised May 4, 2008 and December 16, 2008; accepted January 27, 2009. The associate editor coordinating the review of this paper and approving it for publication was M. Saquib.

C.-X. Wang, X. Hong, and J. S. Thompson acknowledge the support from the Scottish Funding Council for the Joint Research Institute in Signal and Image Processing between the University of Edinburgh and HeriotWatt University, which is a part of the Edinburgh Research Partnership in Engineering and Mathematics (ERPem). The work presented in this paper was also supported partly by the research grant from National Science Council of Taiwan, NSC97-2219-E-006-004. This paper was presented in part at IEEE ICC 2008, Beijing, China, May 2008.

C.-X. Wang is with the Joint Research Institute for Signal and Image Processing, School of Engineering \& Physical Sciences, Heriot-Watt University, UK (e-mail: cheng-xiang.wang@hw.ac.uk).

$\mathrm{X}$. Hong is with the Department of Electrical and Computer Engineering, University of Waterloo, Ontario, Canada (e-mail: x4hong@ecemail.uwaterloo.ca).

H.-H. Chen is with the Department of Engineering Science, National Cheng Kung University, Taiwan (e-mail: hshwchen@ieee.org).

J. S. Thompson is with the Joint Research Institute for Signal and Image Processing, Institute for Digital Communications, the University of Edinburgh, UK (e-mail: john.thompson@ed.ac.uk).

Digital Object Identifier 10.1109/TWC.2009.071075 "borrow" and reuse the licensed spectrum allocated to primary/incumbent systems, under the condition that no harmful interference is caused to the primary services [2]-[5]. The core of a secondary/CR network is a coexistence mechanism which controls the spectrum sharing in such a way that the primary users' operations are not compromised [6]-[8]. A CR user may coexist with the incumbent primary users either on a non-interfering basis [9]-[11] or an interference-tolerant basis [11]-[14]. The former case guarantees the exclusive frequency occupancy for primary users and CR users can only operate in the unused frequency bands, also known as spectrum holes or white spaces [8]. On the other hand, the interference-tolerant case works such that the CR users are allowed to operate on the frequency band assigned to the primary user as long as the total interference power received at the primary user remains below a certain threshold [11]-[14].

Both coexistence mechanisms have pros and cons. The noninterfering based mechanism has so far widely been adopted, e.g., in [9]-[11], due to its advantage that the associated incumbent primary network is not necessarily aware of the existence of the CR network and therefore does not need to be changed. On the other hand, the interference-tolerant based coexistence mechanism requires the primary network to be aware of the CR network and send back the interference levels of all the primary receivers to the CR transmitters. In this case, the feedback mechanism is absolutely essential but sometimes is undesirable for the primary network. Despite the greater implementation challenge, the interference-tolerant based mechanism undoubtedly results in more efficient spectrum utilization than the non-interfering based mechanism. In this paper, we will restrict our studies to interference-tolerant based CR networks.

Capacity analysis is very useful in investigating the ultimate performance limits and thus potential applications of CR systems. Some pioneering information theoretic work on the CR channel capacity were presented in [15], [16]. In particular, for interference-tolerant CR networks, it is necessary to analyze the network capacity under received interference power constraints, which can be specified in terms of either average power (average over fading states) or peak power [14]. An average received interference power constraint is reasonable when the quality of service (QoS) of the primary network is determined by the average signal-to-interferenceand-noise ratio (SINR), e.g., for a delay-insensitive service. When the QoS of the primary network depends on the instantaneous SINR, a peak received interference power constraint is more appropriate [14]. Under an average received interference power constraint at primary receivers, the channel capacities 


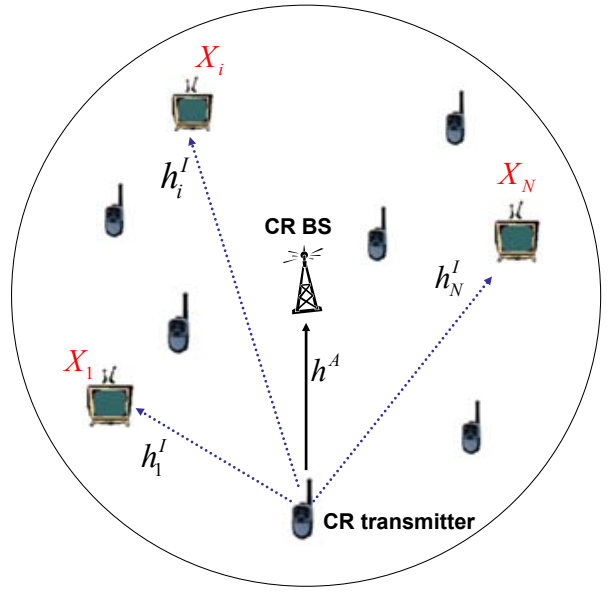

Fig. 1. CR based central access network.

in different AWGN channels in the absence of fading were studied in [12], [13]. In [14], the channel capacities were derived in different fading environments under both average and peak interference power constraints. The above capacity analyses in [12]-[14] are restricted to the link level, where only one CR transmitter opportunistically communicates with one $\mathrm{CR}$ receiver in the presence of a single or multiple primary receivers.

In this paper, we focus on the capacity analysis under average interference power constraints. The contributions made in this paper are two-fold. First, unlike previous studies that are restricted to the link level capacity analysis, this paper investigates the system level capacities of CR networks. We adopt a system model that consists of multiple CR transmitters and multiple primary receivers. Also, the underlying channel models include path loss effects, which preserve location information of the primary/secondary users and therefore allow us to study the impact of the network topology, such as the densities of CR transmitters and primary receivers, on the system capacity. Second, two different CR networks, namely a central access CR network (illustrated in Fig. 1) and a CR assisted virtual multiple-input multiple-output (MIMO) communication network (illustrated in Fig. 2), are analyzed and compared under a common framework. Such a comparison suggests that instead of building pure CR networks that are self-sufficient in delivering competitive wireless services, it is a promising alternative to use CR to assist existing wireless networks to enhance their performance.

The remainder of this paper is outlined as follows. In Section II, the transmit power constraints of CR users are derived when a simple power control scheme is applied. Section III presents a central access CR network and analyzes the uplink channel capacity. In Section IV, a CR assisted virtual MIMO communication network is discussed and its uplink channel capacity is investigated. Finally, conclusions are drawn in Section V.

\section{Power Control And CR Transmit Power CONSTRAINTS}

As illustrated in Figs. 1 and 2, for both CR networks, multiple CR users are assumed to be uniformly distributed in a circular cell with radius $R$. Additionally, we assume that

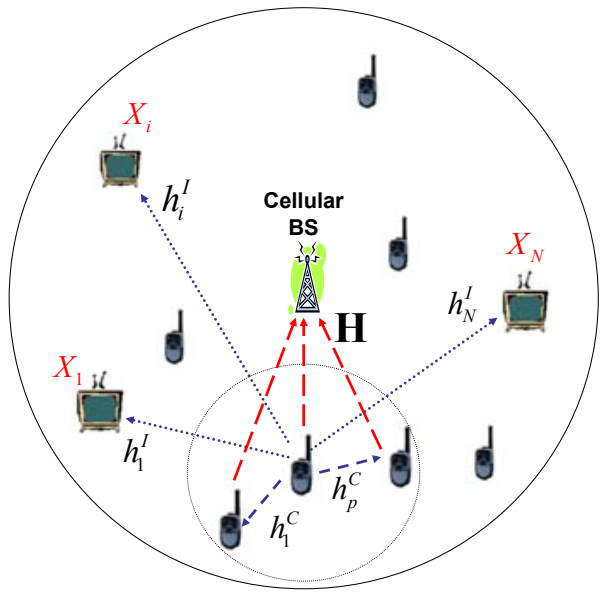

Fig. 2. CR assisted virtual MIMO communication network.

$N$ primary user receivers, denoted as $X_{i}(i=1, \ldots, N)$, are also uniformly distributed in the cell. Within the CR network, we assume that CR users transmit in orthogonal channels to avoid mutual interference. In this paper, we use a time division multiple access (TDMA) scheme, which implies that only one target CR user is scheduled to transmit in a given time slot. Although such a TDMA scheme does not necessarily achieve optimum spectrum efficiency, it leads to a simple and practical power control scheme which will be explained in detail subsequently.

Since only the target CR user is allowed to transmit at any given time, it is the only interference source to the primary network. We refer the underlying channels from the CR transmitter to primary receivers as interference channels. The instantaneous channel power gains from the scheduled $\mathrm{CR}$ transmitter to the $i$ th primary receiver is denoted as $h_{i}^{I}$. In addition, let $P$ be transmit power of the target CR user. Under the average interference power constraints at all $N$ primary receivers, we have $P E\left\{h_{i}^{I}\right\}=P \bar{h}_{i}^{I} \leq I_{0}(i=1, \ldots, N)$, where $E\{\cdot\}$ is the statistical average operator and $I_{0}$ is the maximum average interference power that the primary receivers can tolerate. For analysis simplicity, we assume that the averaged interference channel gain $\bar{h}_{i}^{I}$ within a given time slot can be described by the path loss expressed by [17]

$$
\bar{h}_{i}^{I}=E\left\{h_{i}^{I}\right\}=\frac{\left(h_{c} h_{p}\right)^{2}}{\left(d_{i}\right)^{\alpha}}
$$

where $d_{i}$ is the distance between the target CR transmitter and the $i$ th primary receiver, $\alpha=4$ is the path loss factor, $h_{c}$ and $h_{p}$ are the antenna heights of the CR transmitter and the primary receivers, respectively. In this paper, we assume $h_{c}=h_{p}=1.5 \mathrm{~m}$. We further define

$$
P_{\max }=\frac{I_{0}\left(d_{\min }\right)^{4}}{\left(h_{c} h_{p}\right)^{2}}
$$

as the maximum allowable transmit power, where $d_{\min }=$ $\min \left\{d_{i}\right\}$ stands for the distance between the CR transmitter and the nearest primary receiver. It is noted that $d_{\min } \in$ $[0, R+r]$ always holds, where $r$ is the distance between the target CR transmitter and the cell center. We also assume that the target $\mathrm{CR}$ transmitter can accurately estimate $P_{\max }$, which can be obtained by either listening to a common control 
channel or using certain feedback power control mechanisms [18]. In what follows, we will first derive the probability density function (PDF) $f_{P_{\max }}(x)$ of $P_{\max }$.

As shown in (2), the random variable (RV) $P_{\max }$ is expressed as a function of another RV $d_{\min }$. To calculate $f_{P_{\max }}(x)$, we should first get the cumulative density function (CDF) $F_{d_{\min }}(d)$ of $d_{\min }$, which can be derived from the proposed geometric method detailed in the Appendix. The resulting $\mathrm{CDF} F_{d_{\min }}(d)$ is given by

$$
F_{d_{\min }}(d)=1-\left[\frac{S(d)}{\pi R^{2}}\right]^{N}, \quad 0 \leq d \leq R+r
$$

where

$$
S(d)=\left\{\begin{array}{lr}
\pi R^{2}-\pi d^{2}, & d \in[0, R-r] \\
\pi R^{2}-\pi d^{2}+S_{1}-S_{2}, & d \in\left(R-r, \sqrt{R^{2}-r^{2}}\right] \\
\pi R^{2}-S_{2}-S_{1}, & d \in\left(\sqrt{R^{2}-r^{2}}, \sqrt{R^{2}+r^{2}}\right] \\
S_{2}-S_{1}, & d \in\left(\sqrt{R^{2}+r^{2}}, R+r\right] .
\end{array}\right.
$$

In (4), $S_{1}$ and $S_{2}$ are given by $S_{1}=d^{2}\left(\theta_{1}-\right.$ $\left.\sin \theta_{1} \cos \theta_{1}\right)$ and $S_{2}=R^{2}\left(\theta_{2}-\sin \theta_{2} \cos \theta_{2}\right)$, respectively, with $\theta_{1}=\cos ^{-1}\left|\left(d^{2}+r^{2}-R^{2}\right) /(2 d r)\right|$ and $\theta_{2}=$ $\cos ^{-1}\left|\left(R^{2}+r^{2}-d^{2}\right) /(2 R r)\right|$. The values of $\theta_{1}$ and $\theta_{2}$ are assumed to be in the interval $[0, \pi / 2]$. From (2) and (3), it can easily be shown that the $\mathrm{CDF} F_{P_{\max }}(x)$ of $P_{\max }$ is given by

$$
F_{P_{\max }}(x)=F_{d_{\min }}\left[\left(\frac{h_{c}^{2} h_{p}^{2} x}{I_{0}}\right)^{1 / 4}\right], \quad 0 \leq x \leq P_{\lim }
$$

where $P_{\lim }=I_{0}(R+r)^{4} /\left(h_{c} h_{p}\right)^{2}$ represents the upper limit of $P_{\max }$ when $d_{\min }$ in (2) takes the largest value $R+r$. From (5), the PDF of $P_{\max }$ is given by $f_{P_{\max }}(x)=d F_{P_{\max }}(x) / d x$.

In the following two sections, we will analyze and compare uplink channel capacities of two CR scenarios by assuming that the target CR terminal is always able to transmit with its maximum allowable power $P_{\max }$ determined by the primary network. It should be noted that in practice, the transmit powers of CR terminals are also limited by their own device capabilities. There might be the cases where the primary users are far away from CR users, such that $P_{\max }$ is larger than the realistic transmit power of the CR user constrained by its own device capability. Therefore, the derived results in the following two sections should be considered as upper bounds on the capacity of CR networks without considering practical device limitations.

\section{UPLINK CAPACITY OF A CR BASED CENTRAL ACCESS NETWORK}

In this section, we consider a scenario where CR is used to establish a central access network with a base station (BS) and multiple CR users. The scenario is illustrated in Fig. 1. To communicate with the CR BS, the target CR user transmits at its maximum allowable power $P_{\max }$ based on the TDMA scheme, as described in Section II.

The channel from the CR transmitter to the CR BS is defined as the CR access channel. The underlying instantaneous channel power gain is denoted by $h^{A}$. It follows that the instantaneous uplink channel capacity is given by $C_{C A}=W \log _{2}\left[1+\left(P_{\max } h^{A}\right) / I_{N}\right]$, where $W$ is the signal bandwidth and $I_{N}$ is noise plus interference power at the CR BS. The access channel gain $h^{A}$ can be written as the product of three parts [17]

$$
h^{A}=g_{p}^{A} g_{s}^{A} g_{m}^{A}=\frac{h_{b}^{2} h_{c}^{2}}{r^{4}} g_{s}^{A} g_{m}^{A}
$$

where $g_{p}^{A}, g_{s}^{A}$, and $g_{m}^{A}$ represent the power gains of path loss, shadowing, and multipath fading, respectively. In (6), $r$ is the distance between the CR BS and target CR transmitter, $h_{b}$ and $h_{c}$ are the antenna heights of the CR BS and CR transmitter, respectively. In this paper, we assume $h_{b}=30 \mathrm{~m}$ and $h_{c}=1.5 \mathrm{~m}$. The shadowing factor $g_{s}^{A}$ is a random variable with a log-normal PDF given by $f_{g_{s}^{A}}(x)=\left\{10 \exp \left[-\left(10 \log _{10} x\right)^{2} /\left(2 \delta_{s}^{2}\right)\right]\right\} /\left(\ln 10 \sqrt{2 \pi} \delta_{s} x\right)$, where $\delta_{s}$ is taken to be $8 \mathrm{~dB}$ as a typical value in macrocell environments [17]. We further assume that multipath fading follows a Rayleigh distribution. Correspondingly, $g_{m}^{A}$ follows an exponential distribution and its PDF is given by $f_{g_{m}^{A}}(x)=\left\{\exp \left[-x /\left(2 \delta_{m}^{2}\right)\right]\right\} /\left(2 \delta_{m}^{2}\right)$, where $\delta_{m}$ is the standard deviation of the underlying real Gaussian process and is normalized to $\sqrt{2} / 2$ here. It follows that

$$
C_{C A}=W \log _{2}\left(1+\frac{I_{0}}{I_{N}} \frac{h_{b}^{2}}{h_{p}^{2} r^{4}} d_{\min }^{4} g_{s}^{A} g_{m}^{A}\right)
$$

where $d_{\min }, g_{s}^{A}$, and $g_{m}^{A}$ are independent random variables with PDFs given by $f_{d_{\min }}(x)=F_{d_{\min }}(x) / d x, f_{g_{s}^{A}}(x)$, and $f_{g_{m}^{A}}(x)$, respectively. The ergodic capacity $E\left\{C_{C A}\right\}$ can be calculated numerically using a three-fold integration taken over $d_{\min }, g_{s}^{A}$, and $g_{m}^{A}$.

Fig. 3 shows the normalized ergodic capacity or the spectrum efficiency $E\left\{C_{C A}\right\} / W$ as a function of the primary user number $N$ with $I_{0} / I_{N}=1, R=1000 \mathrm{~m}$, and different values of $r / R$. Corresponding simulation results were also obtained by averaging over 10,000 realizations of the instantaneous capacity calculated from (7). The theoretical results obtained from the numerical integration agree very well with the simulation results. It is clear that given the number of primary users $N$, the ergodic capacity of the uplink CR channel decreases quickly with increasing $r / R$. Given $r / R$, the capacity decreases dramatically as $N$ increases. Only with a small number of primary users $N$, a large capacity can be achieved. This demonstrates that the capacity provided by a CR based central access network is significantly restricted by the number of primary users. As a result, such application is more suitable for less populated rural areas, where the density or number of primary receivers is relatively low.

\section{Uplink CAPACITy of a CR Assisted Virtual MIMO NETWORK}

The second CR scenario we consider in this section is called CR assisted virtual MIMO communication network, as shown in Fig. 2. The purpose of utilizing CR here is to improve the radio access ability of a cellular system. The current and future cellular networks are challenged by users' increasing demand of high quality and high data rate multimedia services. MIMO is envisioned as a key technology to meet this challenge [19]. By deploying multiple antennas at both transmit and receive 


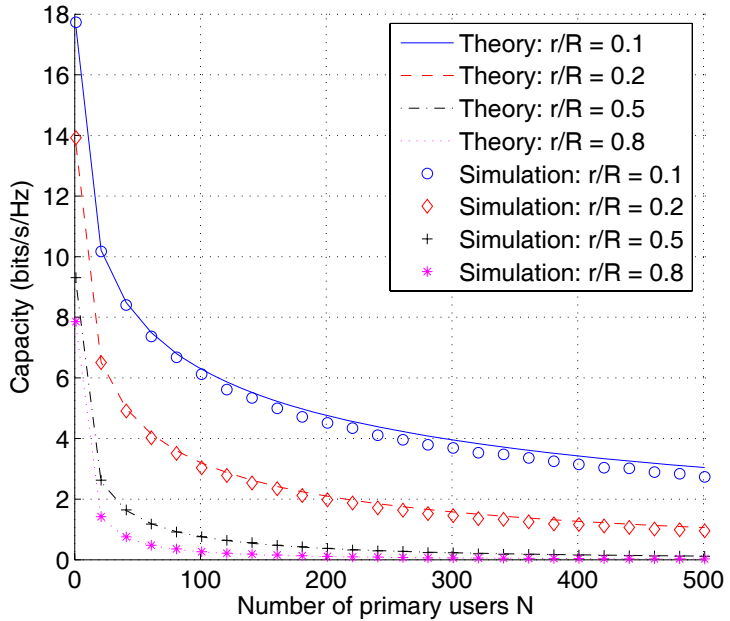

Fig. 3. The uplink normalized ergodic capacity of the CR based central access network as a function of $N$ with different values of $r / R\left(I_{0} / N_{0}=1, R=1000 \mathrm{~m}\right)$.

ends, an MIMO system promises significant capacity increase. However, it is still not feasible to implement a large number of antennas into small-size mobile terminals with sufficient decorrelation among antenna elements [20]. Virtual MIMO communication [21] was proposed as an alternative which emulates an MIMO system by coordinating multiple singleantenna users to form a virtual antenna array (VAA).

In the second CR scenario shown in Fig. 2, a cellular BS equipped with an antenna array is located in the center of a circular cell with radius $R$. The mobile terminals are dualmode devices capable of operating in both cellular bands and CR bands simultaneously. We assume that there are $M$ mobile CR users and $N$ primary users, both uniformly distributed in the cell. The basic idea behind this scenario is to first utilize an ad hoc CR network for helping a target mobile transmitter to cooperate with neighboring mobile terminals in CR bands in order to form a VAA. The VAA will then communicate with the cellular BS antenna array in cellular bands. The constructed CR assisted virtual MIMO system is expected to greatly improve the spectrum utilization efficiency and system capacity.

\section{A. VAA Establishment and Signaling}

The VAA is established in CR bands instead of cellular bands. This can greatly relieve the congestion problem of cellular bands. Once the target transmitter is allocated with the CR bands, it first determines the maximum allowable transmit power $P_{\max }$ given by (2). Then, the CR transmitter broadcasts in the allocated $\mathrm{CR}$ band and cooperates with neighboring users that happen to be inside a circle with radius $\hat{R}$ centered on the CR transmitter. Consequently, the number of cooperating users is a random number, which can be easily derived using basic combinatorial mathematics as a function of $M$ (the number of CR users in the cell), $\hat{R}$, and $R$. Let us denote the number of transmit antenna elements in the VAA as $n_{T}$. We also assume $1 \leq n_{T} \leq 8$ as a constraint imposed by the system design. This means that even there are more than seven other CR users inside the circle, the target
CR transmitter will only cooperate with seven CR users. We arrange the antenna array so that the $p$ th $\left(1 \leq p \leq n_{T}-1\right)$ antenna of the VAA is from the $p$ th cooperating user and the $n_{T}$ th antenna is from the target transmitter.

An uplink transmission is completed in two phases. In the first phase, the symbols are transmitted from the target CR user to $n_{T}-1$ cooperating CR users through the CR channels. The available CR bandwidth $W$ is divided into $n_{T}-1$ channels based on orthogonal frequency division. Also, the maximum allowable power $P_{\max }$ of the target transmitter is allocated to each channel with equal power of $P_{\max } /\left(n_{T}-1\right)$. Let us define the channels from the target user to cooperating users as cooperation channels. The $p$ th cooperation channel gain is denoted as $h_{p}^{C}$, which is given by an equation similar to (6). In the second phase, the cooperating users directly amplify the symbols received in the first phase and retransmit them on the cellular channel [22]. The cellular virtual MIMO channel matrix $\mathbf{H}$ is modeled as the composite of a log-normal shadowing process with a standard deviation of $8 \mathrm{~dB}$ and an independent Rayleigh fading process with a standard deviation of $\sqrt{2} / 2$ for the underlying real Gaussian process.

\section{B. Uplink Virtual MIMO Channel Capacity}

Following similar steps in [23], we can derive the capacity of the virtual MIMO link (in the absence of channel knowledge to the transmitter) as

$$
C_{V M}=\log _{2}\left[\operatorname{det}\left(\mathbf{I}_{n_{R}}+\frac{E_{s}}{\Omega_{0} n_{T}} \mathbf{G}^{-1} \mathbf{H} \mathbf{H}^{H}\right)\right]
$$

where $E_{s} / \Omega_{0}$ is the received SINR at the cellular BS, $\mathbf{I}_{n_{R}}$ denotes a $n_{R} \times n_{R}$ identity matrix, $(\cdot)^{-1}$ gives the inverse of a matrix, $(\cdot)^{H}$ denotes the complex transpose of a matrix, and matrix $\mathbf{G}$ is given by $\mathbf{G}=\mathbf{I}_{n_{R}}+\left[E_{s} /\left(\Omega_{0} n_{T}\right)\right] \mathbf{H} \mathbf{R}_{\hat{n} \hat{n}} \mathbf{H}^{H}$. Here, $\mathbf{R}_{\hat{n} \hat{n}}$ is the covariance matrix of the noise vectors at the VAA given by

$$
\mathbf{R}_{\hat{n} \hat{n}}=\frac{I_{N}}{P_{\max }} \operatorname{diag}\left[\left(h_{1}^{C}\right)^{-1}, \ldots\left(h_{n_{T}-1}^{C}\right)^{-1}, 0\right]
$$

where $I_{N}$ is the received interference plus noise power at the cooperating CR users, diag $[\mathbf{x}]$ returns a square matrix whose diagonal entries are taken from the vector $\mathbf{x}$ while other entries are zero. It should be noted that the classical MIMO channel capacity is given by [19]

$$
C_{M I M O}=\log _{2}\left[\operatorname{det}\left(\mathbf{I}_{n_{R}}+\frac{E_{s}}{\Omega_{0} n_{T}} \mathbf{H H}^{H}\right)\right] .
$$

The comparison of (8) and (10) demonstrates that the virtual MIMO channel capacity differs from the classical MIMO channel capacity only by an additional matrix $\mathbf{G}^{-1}$.

The system parameters that affect the instantaneous channel capacity $C_{V M}$ include the cell radius $R$, cooperation range $\hat{R}$, and value of $I_{0} / I_{N}$. Here, we assume that $R=1000 \mathrm{~m}$, $\hat{R}=20 \mathrm{~m}$, and $I_{0} / I_{N}=1$. Other relevant parameters include the received SNR $E_{s} / \Omega_{0}$ at the cellular $\mathrm{BS}$, the maximum allowable CR transmit power $P_{\max }$, and the VAA antenna numbers $n_{T}$. It is important to mention that the random variables $P_{\max }, n_{T}$, and $\mathbf{H}$ are independent. Taking the mean value of $C_{V M}$ and $C_{M I M O}$ over fading channels $\mathbf{H}$ results in the normalized ergodic virtual MIMO channel capacity 
$E\left\{C_{V M}\right\}$ and real MIMO channel capacity $E\left\{C_{M I M O}\right\}$, respectively.

\section{Numerical Results}

At the link level where a particular user is concerned, both the minimum distance $d_{\min }$ and antenna pairs $n_{T}$ can be treated as fixed parameters. In Fig. 4, we show the numerical results of $E\left\{C_{V M}\right\}$ as a function of $d_{\text {min }}$ with a fixed SNR $E_{s} / \Omega_{0}=8 \mathrm{~dB}$. For $n_{T}=n_{R}>1$, the virtual MIMO channel capacity increases very fast with increasing $d_{\min }$ when $d_{\min }$ is relatively small, e.g., $d_{\min }<150 \mathrm{~m}$. When $d_{\min }$ becomes relatively large, the virtual MIMO channel capacity increases slowly with the increase of $d_{\min }$ and gradually approaches the real MIMO channel capacity. Subsequently, we will fix $E_{s} / \Omega_{0}=8 \mathrm{~dB}$ and further investigate the influence of the number of primary receivers $N$ and the number of CR users $M$ on the channel capacity.

At the system level, both the minimum distance $d_{\min }$ and antenna pairs $n_{T}$ can be treated as random variables whose PDFs are related to the number of primary users $N$ and the number of CR users $M$. The averaged capacities obtained by taking expectation over $d_{\min }$ and $n_{T}$ can serve as a long-term performance indicator of the network. These capacities are shown in Fig. 5, as a function of $N$ with different values of $M$. With the increase of $M$, the virtual MIMO channel capacity increases and gradually approaches the real MIMO channel capacity. This is because that when more CR users are located in the cell, there is a higher probability that more CR users will be within the cooperation range. Consequently, the number of antennas for establishing the VAA will be increased, which will further increase the virtual MIMO channel capacity.

Also, it is noted that the capacity reduction of the CR assisted virtual MIMO channel is not so sensitive to the increase of the number of primary users $N$. This is different from the CR based central access network, the capacity of which decreases dramatically with the increase of $N$, as shown in Fig. 3. On the other hand, the larger number of $\mathrm{CR}$ users $M$ results in a higher virtual MIMO channel capacity. This demonstrates that a $\mathrm{CR}$ assisted virtual MIMO network is more suitable for urban areas, where a high density of CR users exists, despite the fact that the number of primary receivers $N$ might also be large.

\section{CONCLuSions}

In this paper, we have studied the system level capacity of interference-tolerant based CR networks under received average interference power constraints. The proposed CR network consists of multiple primary users and multiple CR users. A simple power control scheme is applied to CR transmitters and a closed-form expression has been derived to specify the $\mathrm{CDF}$ of the maximum allowable CR transmit power.

Two CR scenarios, namely CR based central access network and CR assisted virtual MIMO network, have been studied for potential applications. Numerical and simulation results have shown that the uplink ergodic channel capacity of a CR based central access network is relatively large when the number of primary users $N$ is small, but it decreases rapidly with the increase of $N$. A new expression has been derived for uplink

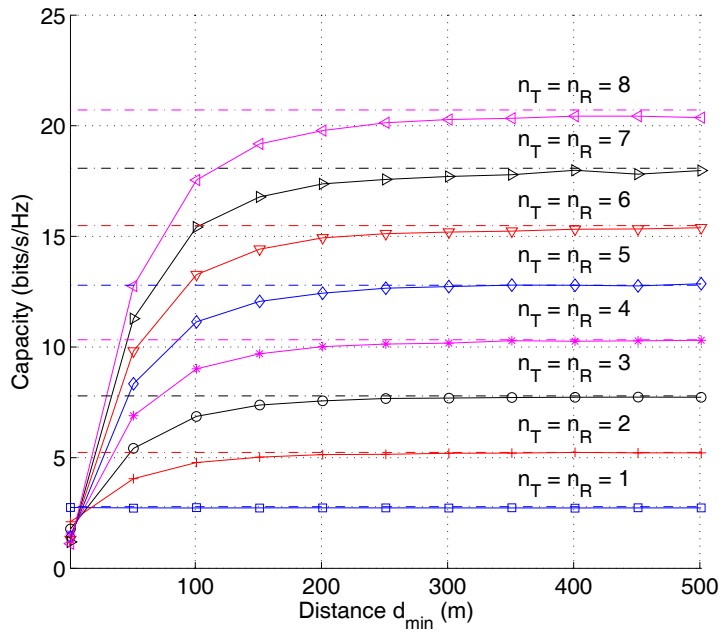

Fig. 4. The uplink normalized ergodic capacity of the $\mathrm{CR}$ assisted virtual MIMO network as a function of $d_{\min }\left(I_{0} / N_{0}=1, E_{s} / N_{0}=\right.$ $8 \mathrm{~dB})$.

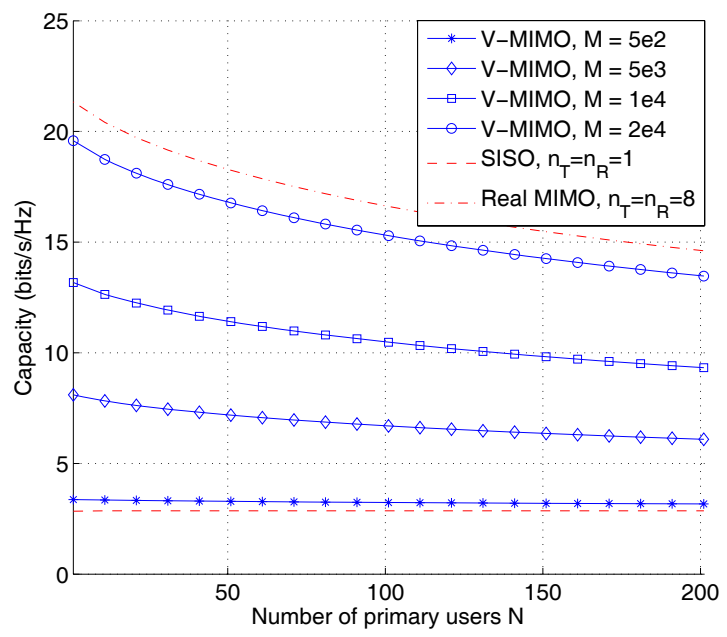

Fig. 5. The uplink normalized ergodic capacity of the CR assisted virtual MIMO network as a function of $N$ with different values of $M\left(I_{0} / N_{0}=1, E_{s} / N_{0}=8 \mathrm{~dB}, \hat{R}=20 \mathrm{~m}, R=1000 \mathrm{~m}\right)$.

channel capacity of a CR assisted virtual MIMO network. It has been demonstrated that the normalized ergodic virtual MIMO channel capacity does not decrease with the increase of $N$ as dramatically as in the first CR scenario. On the other hand, the presence of a very large number of CR users $M$ is crucial to achieve a high channel capacity for the second CR scenario. Our analysis indicates that the CR based central access network is more suitable for less-populated rural areas, while the $\mathrm{CR}$ assisted virtual MIMO communication network performs better in urban environments.

\section{APPENDIX}

\section{DERIVATION OF (3)}

In this appendix, we propose a new geometric method to derive the CDF $F_{d_{\min }}(d)$ of $d_{\min }$ shown in (3). As illustrated in Fig. 6, we use a circular area $C_{1}$ with radius $R$ to represent a cell of the CR network. The center of a cell is denoted as 


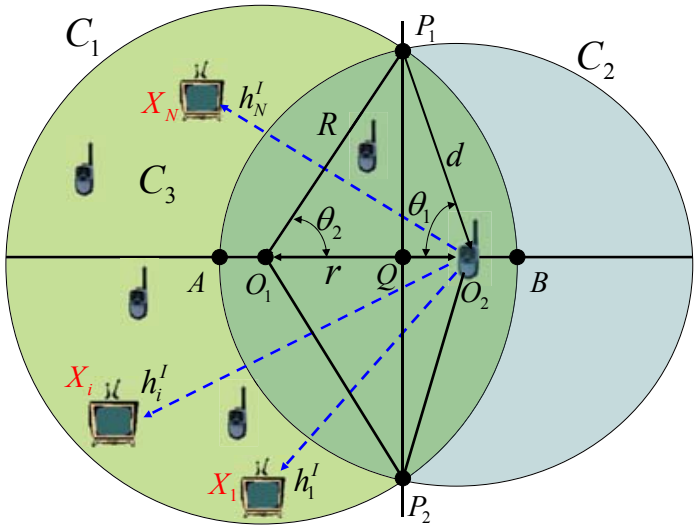

Fig. 6. The proposed geometric method to calculate the CDF $F_{d_{\min }}(d)$ of $d_{\text {min }}$.

$O_{1}$. Multiple CR users and $N$ primary receivers are uniformly distributed within the cell. The target $\mathrm{CR}$ transmitter is located in $O_{2}$ with the distance of $r$ from the cell center $O_{1}$. The CDF $F_{d_{\text {min }}}(d)$ of $d_{\text {min }}$ is the probability that $d_{\text {min }} \leq d$ holds. If we plot another circle $C_{2}$ with the radius $d$ centered on the target CR transmitter $\mathrm{O}_{2}$, the CDF $F_{d_{\min }}(d)$ can be considered as the probability that at least one primary receiver is located within the circle $C_{2}$ and certainly within $C_{1}$. Let us use $S(d)$ to represent the area of the proportion $C_{3}$ that is within $C_{1}$ but outside $C_{2}$. The probability of the random event that all $N$ primary receivers are located within $C_{3}$ can be calculated by $\left[S(d) /\left(\pi R^{2}\right)\right]^{N}$. The probability of the complementary event, i.e., at least one primary receiver is located outside $C_{3}$ but within $C_{1}$, is the $\operatorname{CDF} F_{d_{\min }}(d)$ of $d_{\text {min }}$. It follows that we have $F_{d_{\min }}(d)=1-\left[S(d) /\left(\pi R^{2}\right)\right]^{N}$, as given by (3). The remaining task is to calculate the area $S(d)$ of the proportion $C_{3}$.

It is noted that the radius $d$ of the circle $C_{2}$ is a random variable ranging from 0 to $R+r$. When $d$ increases from 0 to $R+r, S(d)$ will correspondingly decrease from $\pi R^{2}$ to 0 . Only when $d \in[0, R-r], C_{2}$ is completely included in $C_{1}$. If $d>$ $R-r$, the two circles $C_{1}$ and $C_{2}$ will intersect at two points $P_{1}$ and $P_{2}$. The line $P_{1} P_{2}$ is perpendicular to the line $A B$ with the intersection $Q$. When $d \in\left(R-r, \sqrt{R^{2}-r^{2}}\right], Q$ is located between $B$ and $O_{2}$. When $d \in\left(\sqrt{R^{2}-r^{2}}, \sqrt{R^{2}+r^{2}}\right], Q$ is between $O_{2}$ and $O_{1}$. When $d \in\left(\sqrt{R^{2}+r^{2}}, R+r\right], Q$ is between $O_{1}$ and $A$. According to the interval in which $d$ is located, the corresponding area $S(d)$ can be calculated using basic geometric methods. The final result is shown in (4). The derivation of $S(d)$ in (4) for each region of $d$ is quite similar. In what follows, we will only show how to derive $S(d)$ when $d \in\left(\sqrt{R^{2}-r^{2}}, \sqrt{R^{2}+r^{2}}\right]$, i.e., $Q$ is located between $O_{2}$ and $O_{1}$.

As shown in Fig. 6, the triangle $O_{1} O_{2} P_{1}$ has side lengths $r, d$, and $R$. Based on the law of cosines, the angles $\theta_{1}$ and $\theta_{2}$ can easily be calculated. The area of the sector $O_{2} P_{1} A P_{2}$ is given by $2 \pi d^{2} \frac{\theta_{1}}{2 \pi}=d^{2} \theta_{1}$. The area of the triangle $O_{2} P_{1} P_{2}$ is given by $d \sin \theta_{1} d \cos \theta_{1}=d^{2} \sin \theta_{1} \cos \theta_{1}$. The area $S_{1}$ of the segment $P_{1} Q P_{2} A$ is obtained by subtracting the area of the triangle $O_{2} P_{1} P_{2}$ from the area of the sector $O_{2} P_{1} A P_{2}$, i.e., $S_{1}=d^{2}\left(\theta_{1}-\sin \theta_{1} \cos \theta_{1}\right)$. Similarly, $S_{2}$ is the area of the segment $P_{1} Q P_{2} B$, which can be obtained by subtracting the area of the triangle $O_{1} P_{1} P_{2}$ from the area of the sector $O_{1} P 1 B P_{2}$. This results in $S_{2}=R^{2}\left(\theta_{2}-\sin \theta_{2} \cos \theta_{2}\right)$. When $Q$ is located between $O_{2}$ and $O_{1}$, the area $S(d)$ is obtained by subtracting $S_{1}$ and $S_{2}$ from the area of the circle $C_{1}$, i.e., $S(d)=\pi R^{2}-S_{1}-S_{2}$, as shown in (4). This completes the derivation.

\section{REFERENCES}

[1] Federal Communication Commission, Spectrum policy task force report, Washington DC, FCC 02-155, 2 Nov. 2002.

[2] J. Mitola and G. Maguire, "Cognitive radio: making software radios more personal," IEEE Personal Commun. Mag., vol. 6, no. 6, pp. 13-18, Aug. 1999.

[3] C.-X. Wang, H. H. Chen, X. Hong, and M. Guizani, "Cognitive radio network management: tuning in to real-time conditions," IEEE Veh. Technol. Mag., vol. 3, no. 1, pp. 28-35, Mar. 2008.

[4] X. Hong, C.-X. Wang, H. H. Chen, and Y. Zhang, "Secondary spectrum access networks: spatial modeling and system design," IEEE Veh. Technol. Mag., accepted for publication.

[5] H. H. Chen and M. Guizani, Next Generation Wireless Systems and Networks. Chichester, UK: John Wiley \& Sons, 2006.

[6] Federal Communications Commission, Unlicensed operation in the TV broadcast bands, ET Docket No. 04-186, 2004.

[7] Federal Communication Commission, Facilitating opportunities for flexible, efficient, and reliable spectrum use employing cognitive radio technologies, NPRM \& Order, ET Docket No. 03-108, FCC 03-322, 30 Dec. 2003.

[8] S. Haykin, "Cognitive radio: brain-empowered wireless communications," IEEE J. Select. Areas Commun., vol. 3, no. 2, pp. 201-220, Feb. 2005.

[9] IEEE P802.22, Functional requirements for the 802.22 WRAN standard, IEEE 802.22-05/0007r48, 29 Nov. 2006.

[10] S. A. Jafar and S. Srinivasa, "Capacity limits of cognitive radio with distributed and dynamic spectral activity," in Proc. IEEE ICC'06, Istanbul, Turkey, June 2006, pp. 5742-5747.

[11] D. Ugarte and A. B. McDonald, "On the capacity of dynamic spectrum access enable networks," in Proc. IEEE DySPAN'05, Baltimore, USA, Nov. 2005, pp. 630-633.

[12] M. Gastpar, "On capacity under received-signal constraints," in Proc. 42th Annual Allerton Conf. on Commun., Control, and Computing, Oct. 2004, pp. 1322-1331.

[13] M. Gastpar, "On capacity under receive and spatial spectrum-sharing constraints," IEEE Trans. Inform. Theory, vol. 53, no. 2, pp. 471-487, Feb. 2007.

[14] A. Ghasemi and E. S. Sousa, "Capacity of fading channels under spectrum-sharing constraints," in Proc. IEEE ICC'06, Istanbul, Turkey, June 2006, pp. 4373-4378.

[15] N. Devroye, P. Mitran, and V. Tarokh, "Limits on communications in a cognitive radio channel," IEEE Commum. Mag., vol. 44, no. 6, pp. 44-49, June 2006.

[16] S. Srinivasa and S. A. Jafar, "The throughput potential of cognitive radio: a theoretical perspective," IEEE Commun. Mag., vol. 45, no. 5, pp. 73-79, May 2007.

[17] G. L. Stüber, Principles of Mobile Communication, 2nd Edition. Boston, MA: Kluwer Academic Publishers, 2001

[18] S. Kim, H. Jeon, H. Lee, and J. S. Ma, "Robust transmission power and position estimation in cognitive radio," in Proc. Intl. Conf. Inform. Networking (ICOIN'07), Jan. 2007.

[19] A. Paulraj, R. Nabar, and D. Gore, Introduction to Space-Time Wireless Communications. Cambridge University Press, 2003.

[20] C.-X. Wang, X. Hong, H. Wu, and W. Xu, "Spatial temporal correlation properties of the 3GPP spatial channel model and the Kronecker MIMO channel model," EURASIP J. Wireless Commun. and Networking, vol. 2007, article ID 39871, 9 pages, 2007. doi:10.1155/2007/39871.

[21] M. Dohler, "Virtual antenna array," Ph.D. thesis, King's College London, London, UK, 2003.

[22] J. N. Laneman, G. W. Wornell, and D. N. C. Tse, "An efficient protocol for realizing cooperative diversity in wireless networks," in Proc. IEEE Intl. Symp. Inform. Theory, June 2001, pp. 294-298.

[23] F. R. Farrokhi, G. J. Foschini, A. Lozano, and R. A. Valenzuela, "Link-optimal space-time processing with multiple transmit and receive antennas," IEEE Commun. Lett., vol. 5, no. 3, pp. 85-87, Mar 2001. 